\title{
A PRIORITY-BASED ADAPTIVE SCHEME FOR WIRELESS MULTIMEDIA DELIVERY
}

\author{
Edward Casey and Gabriel-Miro Muntean \\ Performance Engineering Laboratory \\ School of Electronic Engineering, Dublin City University, Dublin 9, Ireland \\ Email: \{caseye, munteang\}@eeng.dcu.ie
}

\begin{abstract}
In wireless multimedia streaming, there is a need to allow for client prioritisation in order to enable provision of end user perceived quality in direct relationship with client device importance. Currently, the same priority is given to all clients, independent of their characteristics, often resulting in unfair distribution of throughput. This paper proposes a priority-based wireless adaptive multimedia delivery scheme that enables client prioritisation during multimedia distribution over IP networks. The paper presents simulation results outlining the benefits of applying the algorithm, illustrating the improvement in bandwidth allocation and in overall end user perceived quality. The algorithm focuses on a residential wireless local area network and assigns static priorities based on device characteristics.
\end{abstract}

\section{INTRODUCTION}

Wireless Local Area Networks (WLANs), based on the IEEE 802.11 protocol, have become the popular choice of network infrastructure in the home environment due to their flexibility, mobility and ease of installation. At present residential WLANs are used primarily for Internet browsing and file sharing. However there is a growing trend towards the use of WLANs for the provision of multimedia-based services such as IPTV, VoIP and networked games. This

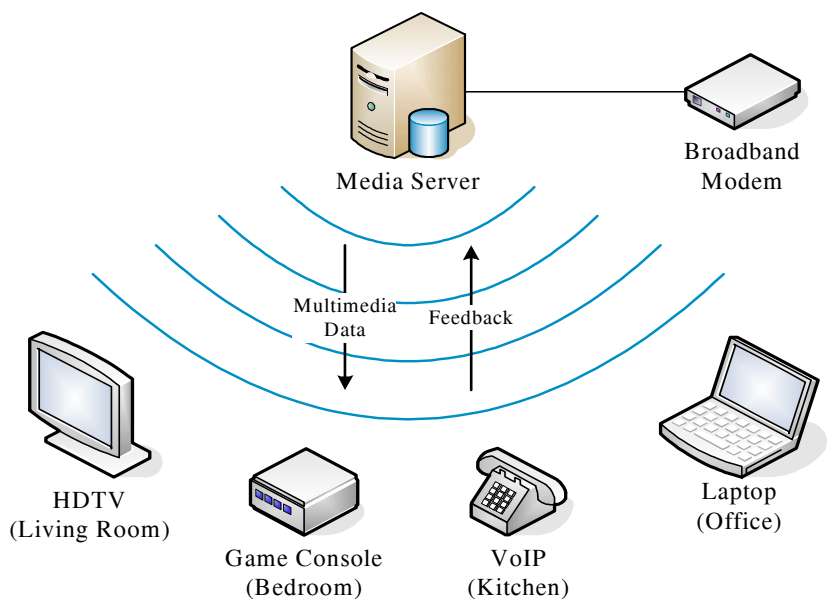

Figure 1 - Typical residential wireless network evolution determines the need for the creation of Media Servers for management and delivery of multimedia content within the home to diverse devices. Multimedia data will be streamed or downloaded to the Media Server via the broadband connection or acquired locally from DVDs, HDDs, etc. where it can be stored until the user wants to view the content. When the user requests selected multimedia content from the Media Server, it will be streamed via the WLAN to the required device. However, the performance of WLANs becomes a crucial issue. Streaming media imposes strict bandwidth, delay and loss bounds on the network. The IEEE 802.11 based WLANs have great difficulty meeting these requirements due to Media Access Control inefficiency and limited shared bandwidth.

Extensive research has proposed different solutions for providing certain levels of Quality of Service (QoS) while streaming multimedia over IP-based networks. It has focused on a congestion control approach to QoS rather than the End User Perceived Quality (EUPQ). EUPQ can be impacted severely in wireless environments due to fluctuations in network conditions, especially when multiple clients stream multimedia simultaneously. As it cannot be guaranteed that all these clients will get highest multimedia quality over WLAN, there is a need to prioritise the multimedia distribution based on the importance of the client. This research is focused on developing an algorithm that identifies device characteristics and based on them and on user subjective assigned importance, adapts and prioritises multimedia streaming in order to increase overall EUPQ. For example a 30-inch High Definition TV (HDTV) requires multimedia content to be streamed at much higher bit rate than a 14 inch portable device, to achieve the same EUPQ

This paper proposes a prioritised adaptation scheme which adapts multimedia content during streaming based on priorities statically assigned by the user to the end device. The scheme adjusts the stream's bit rate to suit available network conditions which in turn affects EUPQ when streaming multimedia. The paper then presents simulation testing results that make use of a basic model which deploys the scheme and delivers multimedia data in different usage conditions using the NS-2 [1] simulator. Conclusions and further work are then presented. 


\section{RELATED WORK}

Extensive research has focused on providing certain level of QoS when streaming multimedia and different approaches were proposed. Based on the layer of the OSI model they are deployed at, these solutions can be classified as adaptive (Application Layer), traffic engineering-based (Network Layer) and access protocol-based (MAC Layer).

Adaptive schemes are the least complex and most flexible mechanisms for providing QoS as they use the existing network infrastructure. Adaptation takes place at the application layer by adjusting the parameters of a multimedia stream to best suit the available network conditions. Source-Based Adaptation requires the sender to adapt the transmission rate of the multimedia stream to match available network bandwidth based on receiver feedback. Rate Adaptation Protocol (RAP), proposed in [2], is a source based TCP friendly Additive Increase Multiplicative Decrease (AIMD) rate adaptation scheme. The TCP-Friendly Rate Control (TFRC) [3] is a congestion control algorithm for unicast traffic. It calculates transmission rate as a function of loss events and round-trip time. Enhanced Loss Delay Adjustment (LDA+) [4] adapts the transmission behaviour of UDP based multimedia streams in accordance with the current network congestion state, whereas the Quality Oriented Adaptation Scheme (QOAS) [5] uses estimated end-user quality in the adaptation loop. Receiver-Based Adaptation Mechanisms allow the receiver to select the quality/rate they wish to receive at. Transcoder based solutions are also available. These schemes match the available bandwidth by transcoding or filtering multimedia streams [6]. Scalable video schemes such as Fine Granular Scalability (FGS) [7] are well suited for transmission in wireless environments, however do not consider other media types illustrated in Figure 1. A comprehensive overview of adaptive techniques can be found in [8] and [9].

Among traffic engineering based solutions, Integrated Services (IntServ) [10] and Differentiated Services (DiffServ) [11] intervene at the Network Layer. IntServ provides QoS guarantees on a per flow basis. DiffServ is a class based approach to QoS which is implemented locally at each router. Unlike IntServ, it does not require setup signalling information to be exchanged prior to transmission. Differentiated services in a wireless environment, requires the MAC to support some degree of separation between different types of services. The IEEE 802.11 MAC does not support this service differentiation. The cost and complexity are particular concerns when implementing the above schemes. It is also difficult to predict end to end behaviour, particularly if all routers are not setup with identical traffic categories.

Access protocols-based solutions include QoS mechanisms for IEEE 802.11 can be placed into three categories; Service Differentiation, Admission Control and Link Adaptation [12]. The original IEEE 802.11 MAC was designed as a best effort network and incorporated limited QoS support in the form of the Point Coordinator Function (PCF) [13]. This allows stations to have priority access to the wireless medium. However, this is an optional access method and is rarely implemented. IEEE 802.11e [14] defines enhancements to the legacy MAC that provide better QoS support using service differentiation. An evaluation of the above mentioned service differentiation schemes can be found in [15] and [16]. Admission control is a QoS mechanism that manages access to the network in order to maintain QoS of existing clients. This scheme is of particular importance in saturated network conditions where the addition of another client would cause failure to support multimedia applications.

Although the above schemes all consider QoS, none of them proposed a prioritised scheme for multimedia delivery in a wireless environment.

\section{PRIORITISED ADAPTIVE MULTIMEDIA STREAMING SCHEME}

\subsection{Problem: Need for prioritisation}

Figure 1 illustrates a typical residential IEEE $802.11 \mathrm{~b}$ WLAN with a number of devices attached. Access to the wireless network is shared equally among these devices resulting in certain QoS targets not being met. For example, consider the situation where two client's, Client 1 is a 28inch HDTV with screen resolution 1920x1080 pixels, requiring at least 4 Mbps video stream to achieve satisfactory EUPQ and Client 2 is a 14-inch TV with screen resolution $1280 \times 720$ pixels which only requires $2 \mathrm{Mbps}$ video stream to achieve the same EUPQ. Both clients request the same video to be streamed from the server via the WLAN. Traditional TCP-Friendly methods such as TFRC will result in both TV's receiving equal share (3 Mbps) of the available bandwidth resulting in Client 1 receiving unsatisfactory EUPQ while Client Two receives video at a quality that may not be appreciated due to its lower resolution display. To overcome this issue a prioritised adaptation scheme is required in order to achieve equal end user satisfaction. These priorities are determined either by objective characteristics such as device properties or by subjective viewer considerations.

\subsection{Solution: Prioritised adaptation scheme}

The proposed scheme describes a method for multimedia delivery in a wireless network using source based rate adaptation with receiver feedback. As discussed in section 3.1, static priorities are assigned based on either device characteristics or subjective viewer assessment in order to maximise overall system EUPQ. An illustration of the systems architecture and operation is shown in Figure 2. The stream originates on the server side of the system where it is stored in the Content Storage. The Transcoding component that transcodes the stream based on the devices 


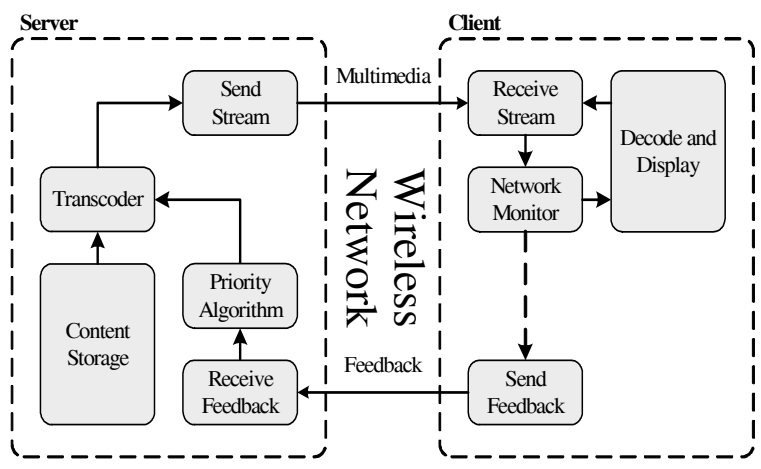

Figure 2 - System architecture and operation

priority and current network condition. This transcoded multimedia stream is then transmitted to the client via the Wireless IP network (IEEE 802.11b for example). On the client side of the system the data is received and then analysed by the Network Monitor component where network statistics such as delay, loss and jitter are continuously recorded and are used for feedback. The received stream is now sent for decoding and display. Feedback is sent periodically to the server where a rate adjustment is requested The server passes this request to a Priority Algorithm component and makes a decision on whether or not the change can be accommodated, based on both the devices priority and the priorities of other devices served by this server over the same network. Its decision is then passed to the Transcoder which performs the required content manipulation. The adjusted stream is now sent to the client and the process is repeated.

The key components of this system are the Server-Side Rate Adaptation Algorithm and the Priority Algorithm. The Rate Adaptation Algorithm makes changes based on network characteristics recorded by the Network Monitor. The adaptation algorithm is compatible with wireless network technology used and optimises the video streaming process, i.e. accounts for wireless transmission errors and controls the transmission rate. The Priority Algorithm receives the rate change request from the Rate Adaptation Module. It is responsible for maintaining priority fairness among devices while maintaining maximum levels of EUPQ. It does so by determining the saturation bandwidth of the wireless network and dividing this bandwidth among connected clients. The bandwidth portion is divided among clients based on their assigned priority. This priority is assigned to individual clients based on their characteristics: e.g. screen resolution, location, user utility, etc.

\section{SIMULATIONS AND RESULTS}

Simulations were carried out using the NS-2 Simulator v2.28. The prioritised adaptation scheme outlined in section 3.2 was implemented by a simulation model and a number of tests were carried out to evaluate the scheme's performance. IEEE $802.11 \mathrm{~b}$ was used for the wireless simulation. A basic client server topology, consisting of one

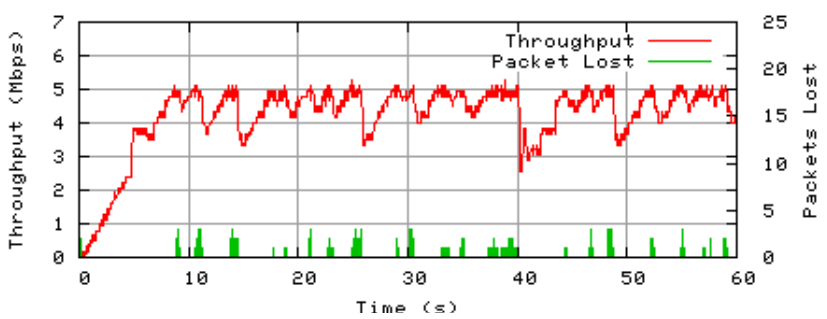

Figure 3 - Single client un-prioritised and prioritised simulation

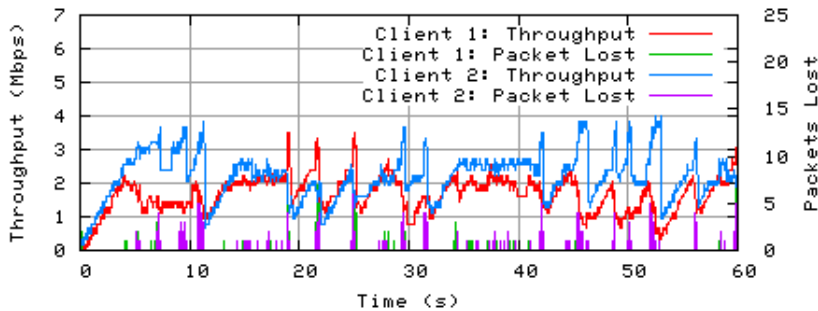

Figure 4 - Two client un-prioritised simulation

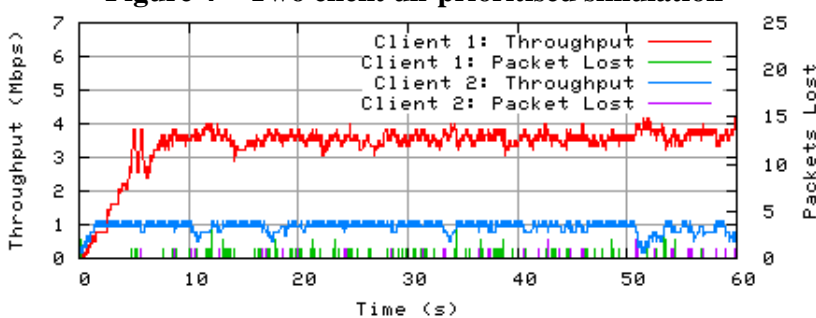

Figure 5 - Two client un-prioritised simulation

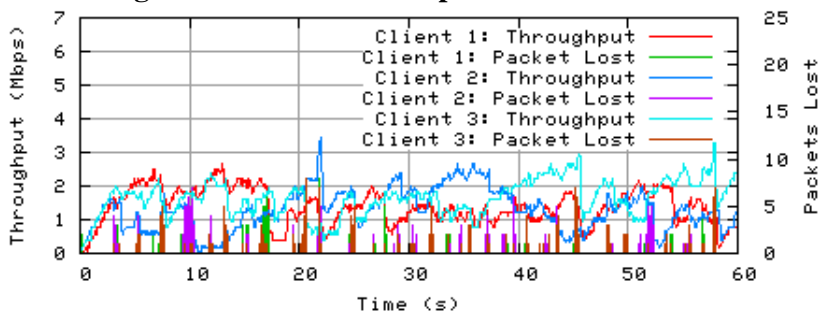

Figure 6 - Two client un-prioritised simulation

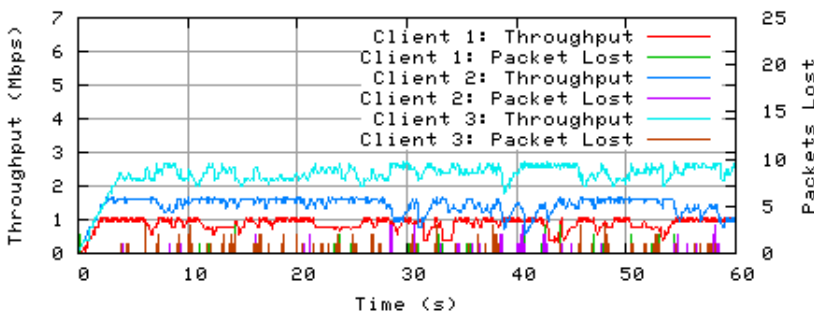

Figure 7 - Two client un-prioritised simulation

to three clients and one server, was used for testing. CBR/UDP multimedia was transmitted using 1000 byte packets. The Rate Adaptation Module used in these simulations was based on a feedback-based AIMD scheme that only considers network loss. They were performed for both the un-prioritised and prioritised cases.

The first test consisted of one client and a server. Multimedia was delivered to the client via the WLAN from the server. Figure 3 shows the results for the un-prioritised and prioritised simulation. Both sets of results were identical as there was no competing source requiring a need for 


\begin{tabular}{||c||c|c||c|c||c|c||}
\cline { 2 - 7 } \multicolumn{1}{c|}{} & \multicolumn{2}{c||}{ Single Client } & \multicolumn{2}{c|}{ Two Clients } & \multicolumn{2}{c|}{ Three Clients } \\
\cline { 2 - 7 } \multicolumn{1}{c|}{} & UP & P & UP & P & UP & P \\
\hline Cli 1 Avg TP & 4.499 & 4.499 & 1.726 & 3.595 & 1.345 & 0.876 \\
\hline Cli 1 Avg Loss & 4.720 & 4.720 & 4.855 & 3.177 & 4.331 & 1.457 \\
\hline Cli 2 Avg TP & - & - & 2.175 & 0.919 & 1.351 & 1.434 \\
\hline Cli 2 Avg Loss & - & - & 5.774 & 0.939 & 4.491 & 1.956 \\
\hline Cli 3 Avg TP & - & - & - & - & 1.675 & 2.403 \\
\hline Cli 3 Avg Loss & - & - & - & - & 4.551 & 2.295 \\
\hline \hline Total Avg TP & $\mathbf{4 . 4 9 9}$ & $\mathbf{4 . 4 9 9}$ & $\mathbf{3 . 9 0 1}$ & $\mathbf{4 . 5 1 4}$ & $\mathbf{4 . 3 7 1}$ & $\mathbf{4 . 7 1 6}$ \\
\hline
\end{tabular}

Table 1 - Average un-prioritised (UP) and prioritised (P) throughput (TP) and loss values for the simulations.

prioritisation. The average throughput obtained when the system reaches steady state is $4.5 \mathrm{Mbps}$, which is about 0.5 Mbps below the theoretical max throughput for a single stream. However, this discrepancy can be attributed to the MAC overheard required to return feedback to the source.

Setup with two clients having different and equal priorities respectively was then implemented. The results are shown in Figure 4 and Figure 5. For exemplification client one is assigned a priority of 20 and client two's priority is 5 . In the equal priority case, both clients receive an average bitrate of about $2 \mathrm{Mbps}$, but both streams experienced severe fluctuations in throughput. In the prioritised case, the throughput of the high priority client has raised to $3.6 \mathrm{Mbps}$, whereas the one of the low priority client has decreased to $0.82 \mathrm{Mbps}$. Both clients receive a steady stream with low loss, resulting in improved EUPQ. A combined average throughput of 4.51 Mbps is obtained, representing a $15.7 \%$ increase compared to the un-prioritised case.

The third test involves a three client setup, again for the equal and different priority cases. Figure 6 shows the equal priority results. Severe fluctuations can be seen, together with high packet loss, which inevitably lead to a lower EUPQ in comparison with the prioritised case shown in Figure 7. For exemplification, clients one to three were assigned priorities 5, 8 and 13 respectively. The Table 1 results show how the proposed scheme achieved expected levels of service differentiation in direct relation with client relative priorities. In this case a significant $7.9 \%$ increase in total throughput in comparison with the equal priority case was recorded.

\section{CONCLUSION AND FURTHER WORK}

This paper proposed a prioritised adaptive scheme for wireless multimedia streaming along with the architecture of the system that deploys it. Its various components and their interaction were explained. Two key components: the server side Rate Adaptation Algorithm and the Priority Algorithm were presented in detail.

Simulation results, carried out in NS2, were shown and the results show a decrease loss and at least a $7.9 \%$ increase in throughput was obtained using the proposed scheme. A reduction in throughput fluctuation was also achieved as well as a degree of prioritised traffic differentiation. All of these factors contribute to an overall improvement in EUPQ.

Future development will incorporate more network and device characteristics to improve the scheme's adaptation accuracy. Also the scheme will be evaluated on the IEEE 802.11g and 802.11e platforms. Objective and subjective assessment of end-user perceived quality is also envisaged.

\section{ACKNOWLEDGEMENTS}

The support of IRCSET Embark Initiative and Enterprise Ireland Commercialisation Fund is gratefully acknowledged.

\section{REFERENCES}

[1] Network Simulator-2, http://www.isi.edu/nsnam/ns/

[2] R. Rejaie, M. Handley and D. Estrin, "RAP: An end-to-end ratebased congestion control mechanism for real-time streams in the internet," Proc. - IEEE INFOCOM. Vol. 3, 1999, pp 1337-1345

[3] M. Handley, S. Flyod, J. Padhye, J. Midmer, “TCP Firendly Rate Control (TFRC): Protocol Specification,” RFC 3448, Jan 2003.

[4] D. Sisalem and A. Wolisz, "LDA+: A TCP Friendly Adaptation Scheme for Multimedia Communication," Proc. of the Int. Conf. on Multimedia \& Expo 3, Vol. 3, Jul - Aug 2000, pp. 1619-1622

[5] G.-M. Muntean, P. Perry and L. Murphy, "A new adaptive multimedia streaming system for all-IP multi-service networks," IEEE Trans. Broadcast., vol. 50, pp. 1-10, 03/. 2004.

[6] A. Vetro, C. Christopoulos and H. Sun, "Video Transcoding Architectures and Techniques: An Overview," IEEE Signal Processing Magazine, Vol. 20, No. 2, Mar. 2003, pp 18 - 29.

[7] H. Radha, M. van der Schaar and Y. Chen, "The MPEG-4 FineGrained Scalable Video Coding Method for Multimedia Streaming over IP," IEEE Trans. on Multimedia, Mar 2001.

[8] D. Wu, Y. T. Hou, W. Zhu, Y.-Q. Zhang, J. M. Peha, "Streaming Video over the Internet: Approaches and Directions", IEEE Trans. On Circuits and Systems for Video Tech., Vol. 11, No. 3, Mar. 2001, pp. 282-300

[9] D. Wu, Y. T. Hou, Y.-Q. Zhang, "Transporting Real-time Video over the Internet: Challenges and Approaches", Proc. IEEE, Vol. 88, No. 12, Dec. 2000

[10] J. Wroclawski, "The use of RSVP with IETF integrated Services," RFC 2210, Sep 1997.

[11] S. Blake, D. Black, M. Carlson, E. Davis, Z. Wang, W. Weiss, "An Architecture for Differentiated Services" RFC 2475, Dec'98

[12] H. Zhu, M. Li, I. Chlamtac and B. Prabhakaran, "A Survey of Quality of Quality of Service in IEEE 802.11 Networks," IEEE Wireless Communications, August 2004.

[13] IEEE "802.11: Wireless LAN Medium Access Control (MAC) and Physical Layer (PHY) specification", 2000.

[14] IEEE 802.11 WG, "Draft Supplement to telecommunications and Information Exchange between Systems LAN/MAN Specific Requirements Part 11: Wireless MAC and PHY Specification: MAC Enhancements for QoS," IEEE 802.11e/draft 11.0 Oct. 2004

[15] A. Lindgren, A. Almquist and O. Schelén, "Quality of Service Schemes for IEEE 802.11 Wireless LANs - An Evaluation," Mobile Networks and Apps., Vol. 8, No. 3, 2003, pp. 223 - 235.

[16] S. Mangold, S Choi, P. May, O. Klein, G. Hiertz and L. Stibor, "IEEE 802.11e Wireless LAN for Quality of Service," Proc. of European Wireless Conf., Florence, Italy, Feb 2002. 